\title{
A 2D Smoothed Particle Hydrodynamics method for liquefaction induced
}

\author{
lateral spreading analysis
}

\author{
Mounir NAILI*, Takashi MATSUSHIMA**, Yasuo YAMADA*** \\ * PhD student, Dept of Eng Mechanics and Energy, University of Tsukuba, Tennodai 1-1-1, Tsukuba 305-8573 \\ * Dr. of Eng., Associate Professor, Dept of Eng Mechanics and Energy, University of Tsukuba, Tennodai 1-1-1, Tsukuba 305-8573 \\ ***Dr. of Eng., Professor, Dept of Eng Mechanics and Energy, University of Tsukuba, Tennodai 1-1-1, Tsukuba 305-8573
}

This paper investigates liquefaction induced lateral spreading using a 2D Smoothed particle hydrodynamics (SPH) based numerical method in the framework of fluid dynamics. Owing to the fact that the induced displacement during lateral spread may reach several meters and the shear strain develops till $100 \%$, a method which is essentially a Langrangian meshfree particle was developed to deal with large displacement. By assuming the liquefied subsoil to behave as a viscous fluid during earthquake shaking, a Bingham type model is used to model the liquefied soil. The ability of the method is checked using results from shaking table experiments to reproduce free surface shape, flow velocity and to investigate the relationship between time history of flow velocity, liquefied soil thickness and surface ground slope.

Key Words: lateral spread, SPH, Bingham model, flow velocity

\section{Introduction}

Liquefaction of loose, saturated, cohesionless soils and other granular material due to earthquake shaking constitute a major source of damage to constructed facilities. For instance, liquefaction induced lateral spreading represents a potential source of distress to piles foundation, waterfront structures and lifelines. During Niigata earthquake (1964) and Kobe earthquake (1995), severe damages were inflicted to pile foundations and waterfront structures.

Lateral spreads at the margins of Port and Rokko Islands caused widespread seaward displacement of caisson walls that severely disrupted port operations. According to Doi and Hamada ${ }^{1)}$, the induced displacement reached several meters in Ohgata area in Niigata city, where the maximum displacement reached over $8 \mathrm{~m}$.

Hence, in order to design structures being able to withstand lateral spreading, the assessment of the induced displacement forms the comerstone to any seismic based design method.

Numerical methods to assess post-liquefaction displacements rely on our understanding of the mechanical behavior of the liquefied soil during shaking. In this respect, undrained monotonic shear tests, shaking table and centrifuge experiments have revealed that the liquefied soil behave either as a solid or a viscous fluid during the course of the loading (Kawakami et al. ${ }^{2)}$, Towhata ${ }^{3)}$, Yasuda ${ }^{4}$ ). Thus, many numerical methods were developed by considering the liquefied soil either as a solid or a fluid. For example, Aydan ${ }^{5)}$ used a FEM formulation to assess the induced post-liquefaction displacement by assuming the soil as a visco-elastic medium. Yasuda et al." proposed a simplified procedure for the analysis of the permanent displacement using the FEM by considering a reduction of the soil stiffness before and after the occurrence of the liquefaction. Assuming the liquefied soil as a Bingham fluid, Uzuoka ${ }^{\text {t) }}$ used the volume of fluid method to predict both the flow process and lateral spreading load. Using the same hypothesis for the liquefied soil, Towhata et al. ${ }^{8)}$ proposed a solution for the permanent displacement on the basis of the minimum energy principle. Using the CIP (Cubic Interpolated pseudopartcile) method, Hadush et al. ${ }^{9)}$ proposed a numerical method for liquefaction induced lateral spreading analysis in the framework of fluid dynamics and were able to simulate lateral spread of a liquefied ground with an overlying non-liquefied layer.

Because induced displacements are large as reported from 
post-earthquake observations, grid based numerical methods such as FEM face sometimes mesh distortion problem as it is difficult to handle large deformation. In this case, special treatment of the mesh is necessary to overcome numerical instability and this may lead to time-consuming computation. Similarly, VOF based on a staggered mesh needs special treatment to track moving boundary and interface. Recently, Maeda and Sakai ${ }^{10)}$ developed a procedure for seepage failure analysis of granular ground using smoothed particle hydrodynamics (SPH).

In this paper, the SPH is investigated in the framework of fluid dynamics to assess ground displacements induced by lateral spreading. The soil is considered as a Non-Newtonian fluid by means of Bingham fluid model. Under this hypothesis, the soil is capable of resisting any shear stress below the yield defined by the residual undrained shear strength. The ability of the method to reproduce free surface shape, time history of flow velocities and its relation to liquefied soil thickness and surface ground slope is investigated.

\section{SPH formulation}

The SPH is essentially a Lagrangian meshfree particle method, where the continuous medium is represented by a set of particles that follow the fluid motion and advect its physical quantities such as mass and momentum. The SPH was originally developed to deal with astrophysical problem and is nowadays applied to treat a variety of problem including fluid flow and solid mechanics.

\subsection{Governing equation}

For a Newtonian incompressible fluid, the shear stress-shear strain rate relationship can be expressed by the following equation:

$$
\tau^{\alpha \beta}=\mu e^{\alpha \beta}
$$

Where $\mu \quad$ :Dynamic viscosity

$e^{\alpha \beta}$ : Deviatoric strain rate tensor.

$$
e^{\alpha \beta}=\frac{\partial v^{\alpha}}{\partial x^{\beta}}+\frac{\partial v^{\beta}}{\partial x^{\alpha}}-\frac{2}{3}(\nabla \cdot \mathbf{v}) \delta^{\alpha \beta}
$$

Where $\mathbf{v}$ : Velocity vector

The motion of an incompressible, uniform density fluid is governed by the conservation of mass and momentum principles. It is expressed by the following two equations.

$$
\begin{aligned}
& \frac{D \rho}{D t}+\rho \frac{\partial v^{\beta}}{\partial x^{\beta}}=0 \\
& \frac{D v^{\alpha}}{D t}=\frac{1}{\rho} \frac{\partial \sigma^{\alpha \beta}}{\partial x^{\beta}}+g^{\alpha}
\end{aligned}
$$

In Eq. (3) and (4), $\rho$ is the density and $g$ is the acceleration of gravity. $\sigma^{\alpha \beta}$ is the total stress tensor which can be written as the sum of an isotropic component $p$ and a viscous component $\tau^{\alpha \beta}$.

$$
\sigma^{\alpha \beta}=-p \delta^{\alpha \beta}+\tau^{\alpha \beta}
$$

Substituting Eq. (5) into the momentum Eq. (4) leads to the equation of motion expressed in the form of:

$$
\frac{D v^{\alpha}}{D t}=-\frac{1}{\rho} \frac{\partial p}{\partial x^{\alpha}}+v \frac{\partial}{\partial x^{\beta}}\left(\frac{\partial v^{\alpha}}{\partial x \beta}\right)+g^{\alpha}
$$

Where: $v=\mu / \rho$ represents the kinetic viscosity.

\subsection{SPH formulation for Navier-Stockes equations}

In SPH a function representing a physical quantity can be written in an integral form as:

$$
f(\mathbf{x})=\int_{\Omega} f\left(\mathbf{x}^{\prime}\right) W\left(\mathbf{x}-\mathbf{x}^{\prime}, h\right) d \mathbf{x}^{\prime} \approx \sum_{j=1}^{N} \frac{m_{j}}{\rho_{j}} f\left(\mathbf{x}_{j}\right) W\left(\mathbf{x}-\mathbf{x}_{j}, h\right)
$$

$h$ represents the smoothing length defining the influence domain and $W\left(\mathbf{x}-\mathbf{x}^{\prime}, h\right)$ the kernel function, which should satisfy the following normalization condition:

$$
\int_{\Omega} W\left(\mathbf{x}-\mathbf{x}^{\prime}, h\right) d \mathbf{x}^{\prime}=1
$$

The derivative of the kernel function is expressed as:

$$
\nabla f(\mathbf{x}) \approx \sum_{j=1}^{N} \frac{m_{j}}{\rho_{j}} f\left(\mathbf{x}_{j}\right) \nabla W\left(\mathbf{x}-\mathbf{x}_{j}, h\right)
$$

In this study, we adopted the quintic spline kernel function having the following expression:

$W(r, h)=\alpha_{D} \begin{cases}(3-s)^{5}-6(2-s)^{5}+15(1-s)^{5} & \text { if } 0 \leq \mathrm{s}<1 \\ (3-s)^{5}-6(2-s)^{5} & \text { if } 1 \leq \mathrm{s}<2 \\ (3-s)^{5} & \text { if } 2 \leq \mathrm{s}<3 \\ 0 & \text { if } \mathrm{s} \geq 3\end{cases}$

Where $r$ is the distance between the particles, $s$ is equal to $r / h$ and $\alpha_{D}$ is a normalization constant having the values of $1 / 120 \mathrm{~h}$, $7 / 478 \pi h^{2}$ and $3 / 359 \pi h^{3}$ in one, two and three-dimensional space respectively. In this study, the smoothing length $h$ is taken as 1.1 times the initial spacing between particles.

The SPH formulation for Eq. (3) and Eq. (6) can be achieved using Eq. (7) and Eq. (9). After transformation we get:

$$
\frac{D \rho_{i}}{D t}=\sum_{j=1}^{N} m_{j}\left(v_{i}^{\beta}-v_{j}^{\beta}\right) \frac{\partial W_{i j}}{\partial x_{i}^{\beta}}
$$




$$
\frac{D v_{i}^{\alpha}}{D t}=\sum_{j=1}^{N} m_{j}\left(\frac{\sigma_{i}^{\alpha \beta}}{\rho_{i}^{2}}+\frac{\sigma_{j}^{\alpha \beta}}{\rho_{j}^{2}}\right) \frac{\partial W_{i j}}{\partial x_{i}^{\beta}}+g^{\alpha}
$$

From Eq. (11), Roman subscripts $i j$ are used to denote the particles identification; while Greek indices $\alpha \beta$ are used to express the coordinate system. Therefore, in Eq. (11) and (12), $W_{i j}$ represents the smoothing function of particle $i$ evaluated at particle $j$.

Using Eq. (5) for the total stress, Eq. (12) can be expressed as:

$$
\begin{aligned}
& \frac{D v_{i}^{\alpha}}{D t}=-\sum_{j=1}^{N} m_{j}\left(\frac{p_{i}}{\rho_{i}^{2}}+\frac{p_{j}}{\rho_{j}^{2}}\right) \frac{\partial W_{i j}}{\partial x_{i}^{\alpha}}+ \\
& \sum_{j=1}^{N} m_{j}\left(\frac{\mu_{i} \varepsilon_{i}^{\alpha \beta}}{\rho_{i}^{2}}+\frac{\mu_{j} \varepsilon_{j}^{\alpha \beta}}{\rho_{j}^{2}}\right) \frac{\partial W_{i j}}{\partial x_{i}^{\beta}}+g^{\alpha}(13)
\end{aligned}
$$

In Eq. (13), the first part of the right hand side represents the SPH approximation for the pressure term, while the second part represents the viscous forces. The pressure terms are computed using an appropriate equation of state which relates the hydrostatic pressure to local densities. Monaghan ${ }^{11)}$ suggested the following equation of state while modeling free surface problem.

$$
p=B\left(\left(\frac{\rho}{\rho_{0}}\right)^{\gamma}-1\right)
$$

Where $\gamma$ is a constant and it is taken equal to 7 in this study. $\rho_{0}$ represents the initial density while $B$ is a parameter that sets a limit for the maximum change of the density. In order to limit the density fluctuation to an acceptable value of $1 \%$, the value of the sound speed should be appropriately chosen.

\section{Extension of SPH to model lateral spread}

In this study, the SPH method is formulated in the framework of fluid dynamics. Hence, the liquefied soil is assumed to behave as a viscous fluid during shaking.

In order to extend the SPH method to model lateral spread, an appropriate viscosity coefficient should be defined for liquefied soil. In this respect, viscosity of liquefied soil was investigated using shaking table experiments Towhata ${ }^{12)}$, Hamada and Wakamatsu $^{13)}$, dropping ball method Miyajima et al. ${ }^{14)}$, steel ball pull-up test and viscometer Kawakami et al. ${ }^{2}$.According to the obtained experimental results, it was found that the viscosity coefficient of the liquefied soil decreases with increasing shear strain rate. This suggests that the behavior of the liquefied soil could be assumed as a non-Newtonian fluid. Based on the results obtained from viscometer, Uzuoka $^{7)}$ proposed a relationship between the shear strain rate and the shear stress in the form of a Bingham model. Accordingly, this study adopts the concept of a Bingham model to extend the SPH method to a non Newtonian fluid as it will be discussed in the next section.

\subsection{SPH formulation for a non- Newtonian fluid}

The general equation of the momentum in Eq. (13) is used to model a Non-Newtonian fluid. The main idea consists in defining an equivalent viscosity to be incorporated in the SPH approximation of the viscous forces.

According to the concept of the Bingham model, the soil is capable of resisting any shear below the yield stress represented by the minimum undrained shear strength and then behaves like a Newtonian fluid for shear stresses greater than the yield stress. A Bingham type fluid has the following shear stress-shear strain rate relationship.

$$
\tau=\tau_{\min }+\mu_{B} \dot{\gamma}
$$

Where $\tau_{\min }$ represents the minimum undrained shear strength and $\mu_{\mathrm{B}}$ its viscosity

If we define an equivalent viscosity in the form of Eq. (16), a non Newtonian fluid can be modeled as a Newtonian type fluid.

$$
\mu_{e f f}=\mu_{B}+\frac{\tau_{\min }}{\dot{\gamma}}
$$

Where $\gamma$ is given by the second invariant of the deviatoric strain rate tensor.

$$
\gamma=\sqrt{\frac{1}{2} e^{\alpha \beta} e^{\alpha \beta}}
$$

\section{Numerical simulations}

In this section, the SPH method previously described will be validated using results obtained from $1 \mathrm{~g}$ shaking table experiments. The first part of the numerical simulation attempts to reproduce the final free surface shape and the velocity flow obtained from a lateral spread experiment conducted by Hamada et $\mathrm{al}^{15}$ ). The second part of the numerical simulations aims to investigate the relationship between the flow velocity with respect to the soil thickness and the slope gradient, and tind out how the shape of the time history of the flow velocities do change with the soil layer thickness.

\section{1 Lateral spreading experiment}

To investigate the characteristics of the ground movement after the earthquake motion ceases, Hamada et al. ${ }^{15)}$ conducted a series of $1 \mathrm{~g}$ shaking table experiments in which a model ground with an initial plane surface was constructed in a rigid soil box. The model has $3 \mathrm{~m}$ long, $1 \mathrm{~m}$ wide and $0.3 \mathrm{~m}$ high. The box was vibrated in the lateral direction until a total liquefaction occurs, and then lifted with a specific gradient using a hydraulic jack. During the experiment the final shape of the free surface was continuously monitored and the time history of the induced 
displacements at 3 different depths in the central section of the box was measured. Different tests were performed by varying the initial relative density and the inclination of the soil box. Figure 1 illustrates the experimental setup.

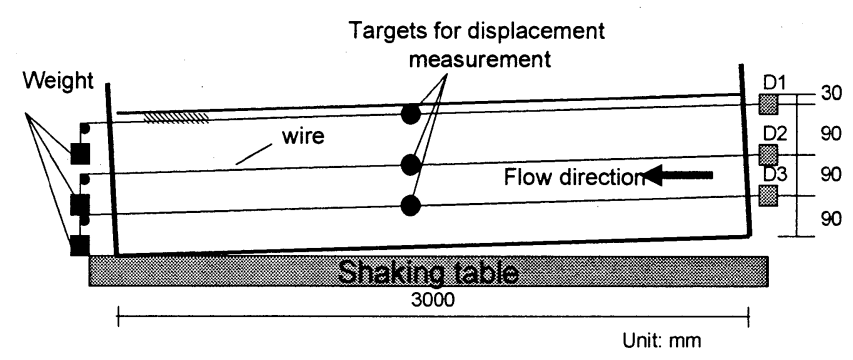

Fig. 1 Outline of the experimental setup ${ }^{15)}$

In a previous study, Naili et al. ${ }^{16)}$ carried out numerical simulations to assess the time history of the induced displacement at three different locations at the central cross section, and compared the results with the experimental observations.

In this simulation, we attempt to reproduce the observed free surface and flow velocity of the experiment in which the soil box inclination $\theta=4.2 \%$. To achieve this, the minimum undrained shear strength and the viscosity coefficient constitute a prerequisite. According to the experimental observation, the displacement of the liquefied soil was triggered when the slope reached an inclination $\theta=2 \%$. Accordingly, the minimum undrained shear strength $\tau_{\min }$ was computed at the middle of the soil deposit as well as at the bottom. Moreover, taking into account the experimental results on the characteristics of the liquefied soil, the viscosity coefficient was varied from $0.01 \mathrm{~Pa} \cdot \mathrm{s}$ to $10 \mathrm{~Pa} \cdot \mathrm{s}$. The minimum undrained shear strength can be assessed considering the equilibrium forces of a sloped ground with an inclination $\theta$. The resistant shear strength needed to maintain the slope into equilibrium represents the minimum undrained shear strength. This can be expressed by $\tau_{\min }=\not h \cos (\theta) \sin (\theta)$, where $\gamma$ is the saturated unit weight of the soil equal to $1800 \mathrm{kN} / \mathrm{m}^{3}$ in this study and $h$ represents the soil height at which $\tau_{\mathrm{min}}$ is assessed.

Table 1 bellow summarizes the numerical parameters used during the simulations.

Table 1 Numerical parameters used during simulations

\begin{tabular}{|c|c|c|}
\hline Cases & Viscosity (Pa.s) & $\tau_{\min }(\mathrm{Pa})$ \\
\hline 1 & 1.0 & 54 \\
\hline 2 & 0.1 & 54 \\
\hline 3 & 0.01 & 54 \\
\hline 4 & 10.0 & 54 \\
\hline 5 & 1.0 & 108 \\
\hline
\end{tabular}

\section{(1) Numerical setup}

A total number of 2250 material particles uniformly distributed, equally spanning the soil box were used to represent the model ground with a surface gradient $\theta=4.2 \%$. Particles with Lennard-Jones potential ${ }^{11)}$ were employed to represent a non slip boundary conditions at the wall surface. Figure 2 shows the numerical setup at the beginning of the calculation process.

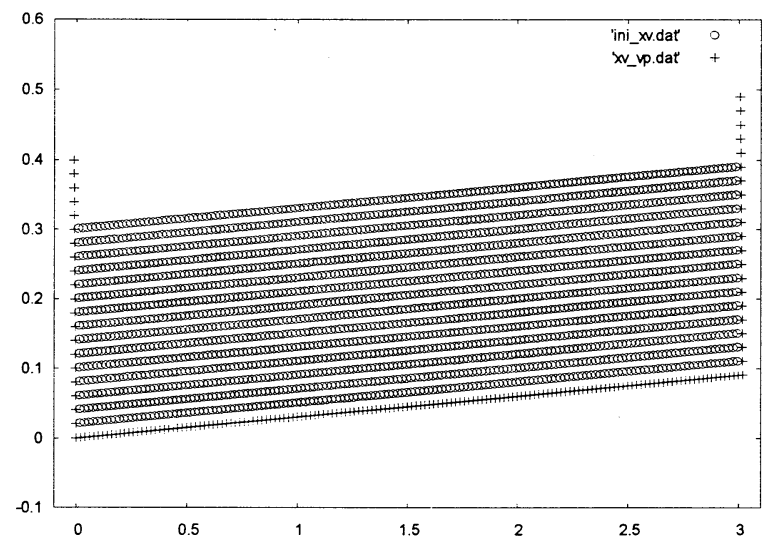

Fig. 2 Initial condition at $t=0$ s.

\section{(2) Simulations results}

To satisfy the CFL (Courant-Fredrich-Levy) condition, a time step $\Delta \mathrm{t}=10^{-4} \mathrm{~s}$ was adopted throughout the calculation process. However, it was noticed that shortly after the start of the computation, a sudden blowup of the particles occurred in the calculation domain. To overcome this numerical instability, we used the XSPH technique proposed by Monaghan ${ }^{17)}$ where the particles move according to Eq. (18).

$$
\begin{aligned}
& \frac{d x_{i}}{d t}=v_{i}-\varepsilon \sum_{j} \frac{m_{j}}{\rho_{j}} v_{i j} W_{i j} \\
& v_{i j}=v_{i}-v_{j}
\end{aligned}
$$

Where $\varepsilon$ is a constant in the range $0 \leq \varepsilon<1$. This technique states that each particle moves in a velocity closer to the average velocity of the neighboring particles. By conducting several simulations and comparing the simulated free surface shapes with the observed one, a value of $\varepsilon$ equal to 0.5 seems to give acceptable and comparable results as shown in figures 3 and 4 .

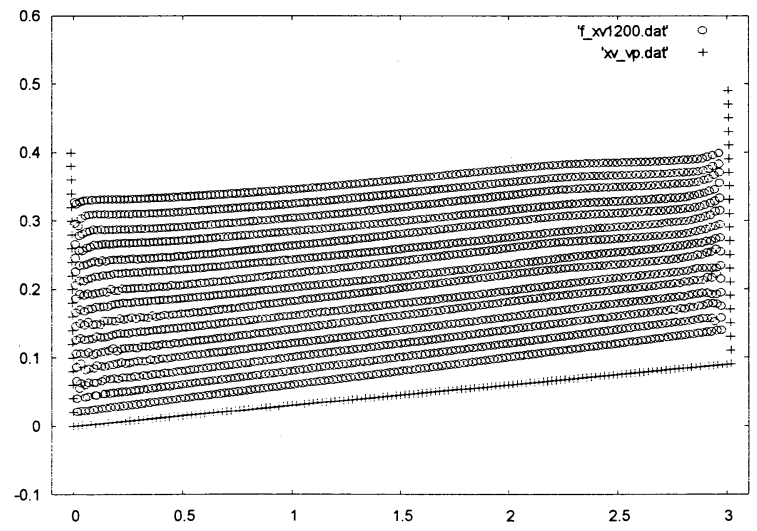

Fig. 3 Final free surface shape at $t=12 \mathrm{~s}$. 


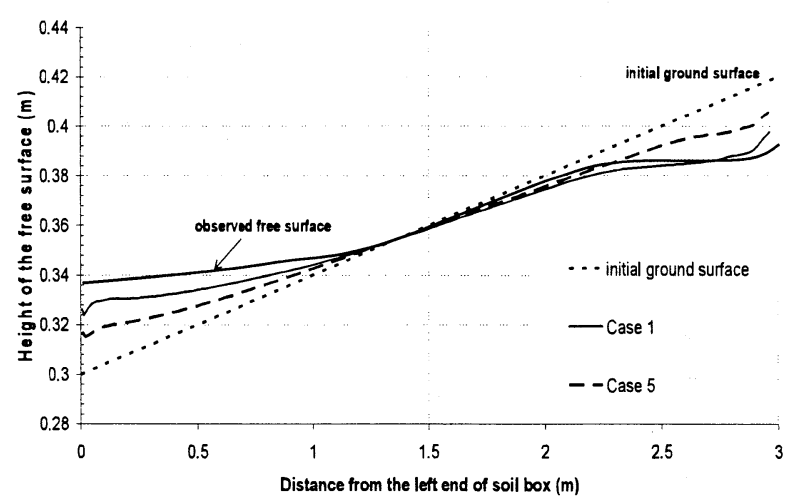

Fig. 4 Simulated free surface for case 1 and 5.

The simulated free surface for case 1 and 5 as seen in figure 4 reproduced clearly the trend of the observed free surface during the experiment. By representing the vectors displacement of the free surface, we notice that the upstream part subsided due to gravity while the downstream heaved due to the volume transfer from the upstream. The central part kept almost the same configuration as the initial ground surface. Figure 5 shows the current observed feature of the free surface in case 1 .

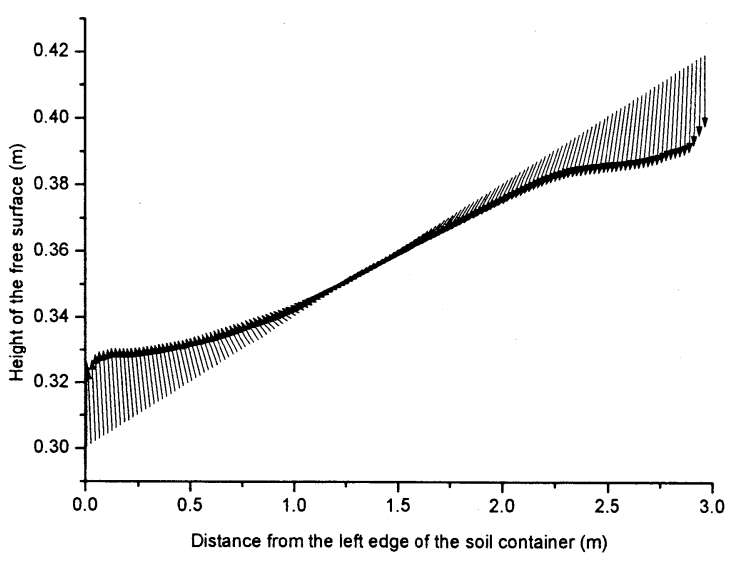

Fig. 5 Vector displacement at the top of the free surface for case 1.

Table 2 below summarizes the maximum flow velocities observed during the experiment.

Table 2 Experimental observed maximum flow velocities ${ }^{15}$

\begin{tabular}{|c|c|}
\hline $\begin{array}{c}\text { Height } \\
(\mathrm{cm})\end{array}$ & $\begin{array}{c}\text { Maximum flow velocity } \\
(\mathrm{cm} / \mathrm{s})\end{array}$ \\
\hline 3 & 0.01 \\
\hline 12 & 0.03 \\
\hline 21 & 0.06 \\
\hline
\end{tabular}

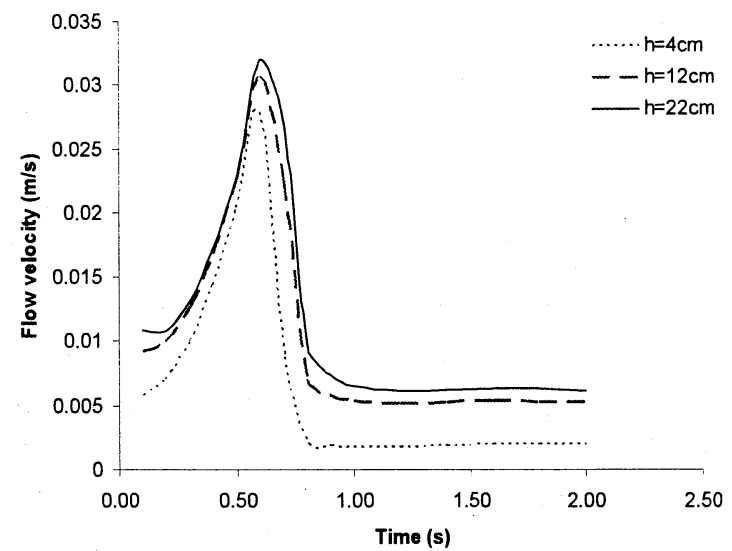

Fig. 6 Time history of flow velocity for case1.

Figure 6 depicts the time history of the flow velocity during the first $2 \mathrm{~s}$ of the simulation at the central part of the soil container at three different depths for case 1 . The following observations can be made:

- The simulated maximum flow velocity for case 1 is smaller than the observed one during the experiment as shown in Table 2.

- The velocities decrease slowly after the steady state is reached and do not vanish as in the experiment. This is due to the fact that the model does not take into consideration the pore water pressure dissipation and the recovery of the soil rigidity.

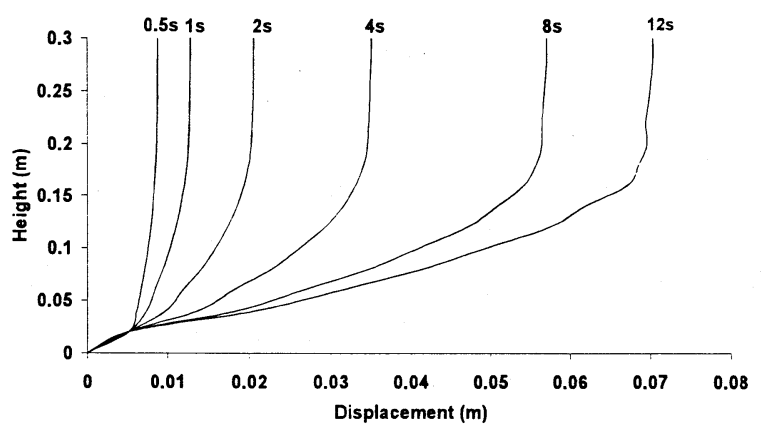

Fig. 7 Displacement distribution along the height at the central cross section for cases 1 and 4.

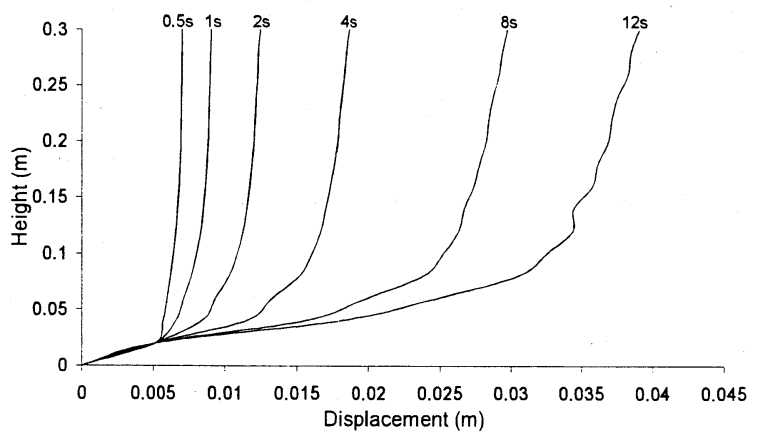

Fig. 8 Displacement distribution along the height at the central cross section for case 5 . 
Figures 7 and 8 show the displacement distribution along the height at the central cross section of the liquefied layer for case 1 , 4 and 5 respectively. It can be seen that the induced displacements are insensitive to the yield viscosity coefficient. This can be explained by the fact that the induced shear strain rate throughout the simulation is smaller than $1 / \mathrm{s}$, which according to Eq. (15) gives almost the same values of the equivalent viscosity, and thus same range of the induced displacement. However, the displacements respond strongly to any change in the minimum undrained shear strength, as in case 5 where the induced displacements are half of the simulated one for cases 1 and 4 .

In both cases 1 and 4, the induced shear strain is of the order of $20 \%$ which is in the range of the observed value during earthquake.

\section{2 Numerical simulation of flow velocity with respect to surface slope gradient and liquefied layer thickness}

In their investigation using shaking table test, Hamada and Wakamatsu ${ }^{13)}$ conducted experiments to clarify the mechanism of liquefaction-induced lateral spreading and find out the nature of the liquefied soil. They also focus their research on predicting the magnitude of the induced lateral displacements. They stated that the liquefied soil behaves as a viscous fluid during shaking and as a solid after the dissipation of the pore water pressure. Accordingly, the Reynolds similitude law encountered in fluid dynamics can be applied between models and actual grounds.

By running several experiments of a sloped ground model with different layer thickness ranging between 15 and $38 \mathrm{~cm}$ and surface gradient varying between 1 and $5 \%$ prepared in a soil box of $3 \mathrm{~m}$ long and $1 \mathrm{~m}$ wide, Hamada and Wakamatsu showed that the velocity of the ground flow is proportional to the square root of the layer thickness. By assuming a flow velocity distribution as onc fourth of a sine wave, they proposed a relationshin for the maximum velocity at the ground surface with respect to soil layer thickness, ground slope and viscosity of the liquefied layer.

In this section, we do not intend to simulate the experiment performed by Hamada and Wakamatsu, but rather use the experimental setup described in section (4.1) to investigate numerically the relationship between the shapes of the time history of the flow velocity, the liquefied layer thickness and the ground gradient slope.

We conducted numerical simulations using liquefied layer thickness ranging from $16 \mathrm{~cm}$ to $50 \mathrm{~cm}$ with a ground gradient slope of $3 \%$ and $5 \%$. We attempt to focus on how the maximum velocity and the required time to reach this maximum do very with layer thickness and ground gradient slope.

\section{(1) Numerical setup}

Total 10 cases of simulations were performed considering a liquefied ground model of thickness varying between $16 \mathrm{~cm}$ and $50 \mathrm{~cm}$ with a ground slope of $3 \%$ and $5 \%$ inside a soil box of a $3 \mathrm{~m}$ length.
The Bingham model is taken as the constitutive law for the liquefied ground considering a yield viscosity $\mu$ of $1 \mathrm{~Pa} \cdot \mathrm{s}$.

The minimum undrained shear strength was assessed for each case at the middle of the soil layer. Figure 9 shows the computed minimum undrained shear strength for each liquefied layer thickness.

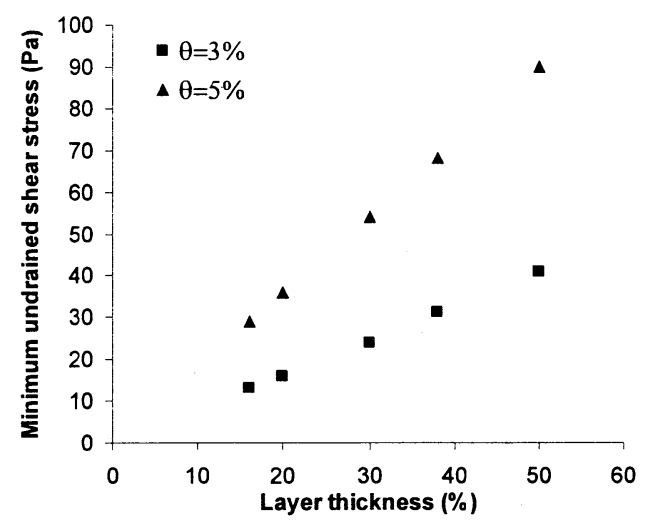

Fig. 9 Minimum undrained shear stress versus layer thickness

\section{(2) Simulations results}

Numerical simulations showed that the maximum flow velocity occurs within the first $2 \mathrm{~s}$ of the time calculation. Accordingly, for better resolution, the time history of flow velocity is plotted during this time span.

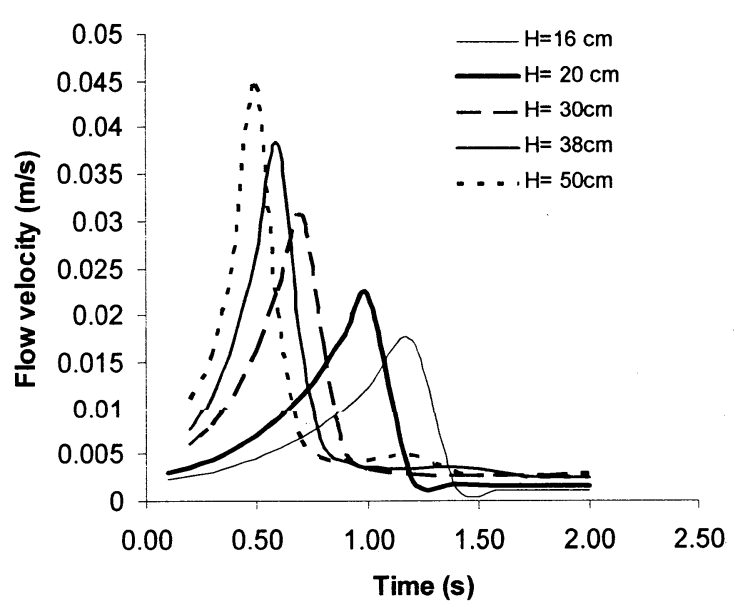

Fig. 10 Time history of flow velocity for slope gradient $\theta=3 \%$

Figures 10 and 11 depict the time history of the flow velocities for a surface ground slope $\theta$ equal to $3 \%$ and $5 \%$ respectively.

It can be seen that the shape of the curve at its base widens with decreasing liquefied soil thickness while the velocity flow increases with increasing soil thickness. However, the time span necessary to reach the maximum velocity is longer for shallow liquefied soil layers.

Generally, it is observed that the induced maximum flow velocity is larger for thick liquefied soil layer which is in agreement with physical and experimental observations. 
Moreover, both graphs illustrate clearly that the peak of the flow velocity for each curve lies on an envelope curve that decays in an exponential way with increasing time.

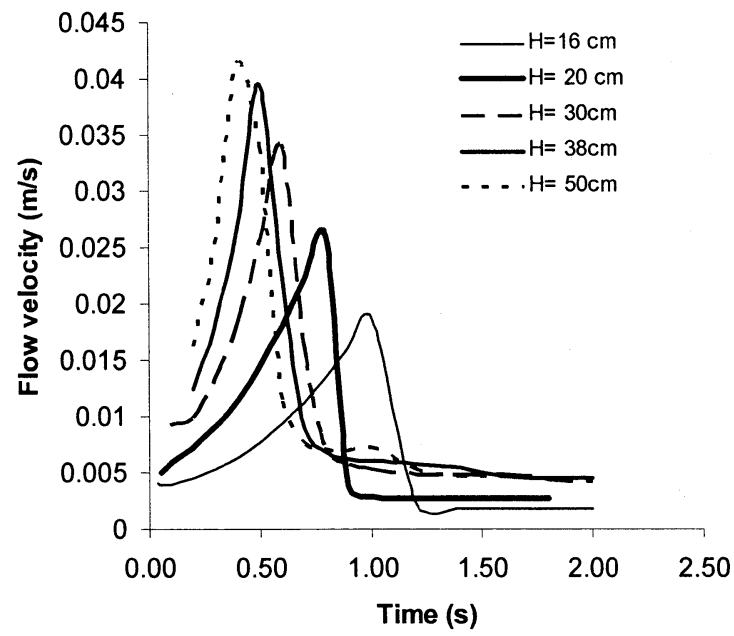

Fig. 11 Time history of flow velocity for slope gradient $\theta=5 \%$

\section{(3) Regression analysis}

The results of the simulations showed that there is a relationship between the maximum flow velocity and the liquefied soil thickness.

A similar relationship exists also between the time required to reach the maximum flow velocity and the liquefied layer thickness. Figure 12 expresses this relationship for a slope gradient $\theta=3 \%$ and $5 \%$.

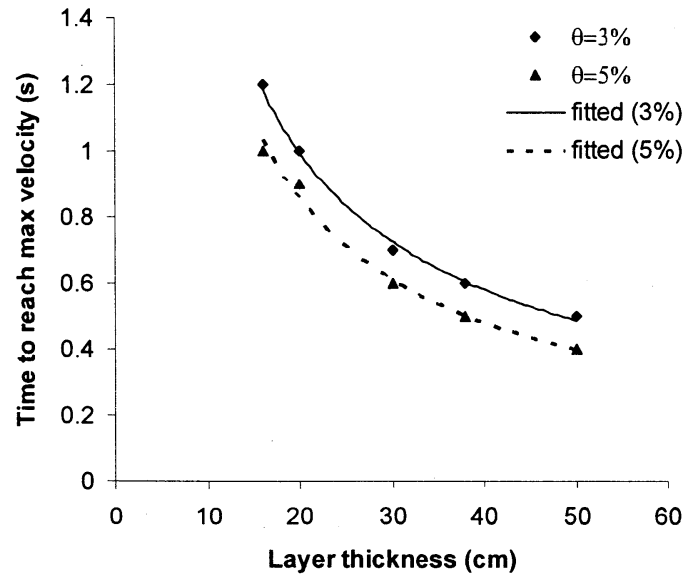

Fig. 12 Time to reach maximum velocity versus liquefied soil thickness

Regression analysis to express the relationship between the time span needed to reach the maximum flow velocity and the corresponding liquefied soil thickness was derived.

The best fit that expresses this relationship is given by Eq. (19) and Eq. (20) respectively for slope gradient $\theta=3 \%$ and $\theta=$ $5 \%$.

$$
\begin{aligned}
& T=10.22(H)^{-0.78} \\
& T=10.41(H)^{-0.83}
\end{aligned}
$$

In the same manner, the relationship between the maximum flow velocities and the liquefied layer thickness is illustrated in figure 13 for a slope gradient of $3 \%$ and $5 \%$.

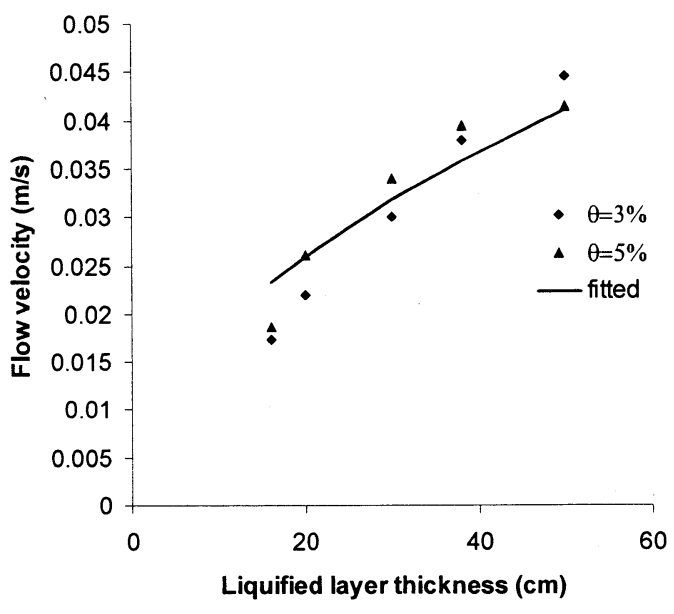

Fig. 13 Flow velocity versus liquefied layer thickness

Similarly, regression analysis was performed to express the relationship between the flow velocity and the square root of the liquefied soil thickness. This relationship is given by Eq. (21).

$$
V=0.58 H^{0.5}
$$

\section{(4) Time history of surface displacement}

The time history of the induced displacement at the surface of the liquefied layer was also assessed at the central cross section for a slope gradient $\theta=3 \%$ and $5 \%$. Figures 14 and 15 depict the corresponding time history for each liquefied layer thickness.

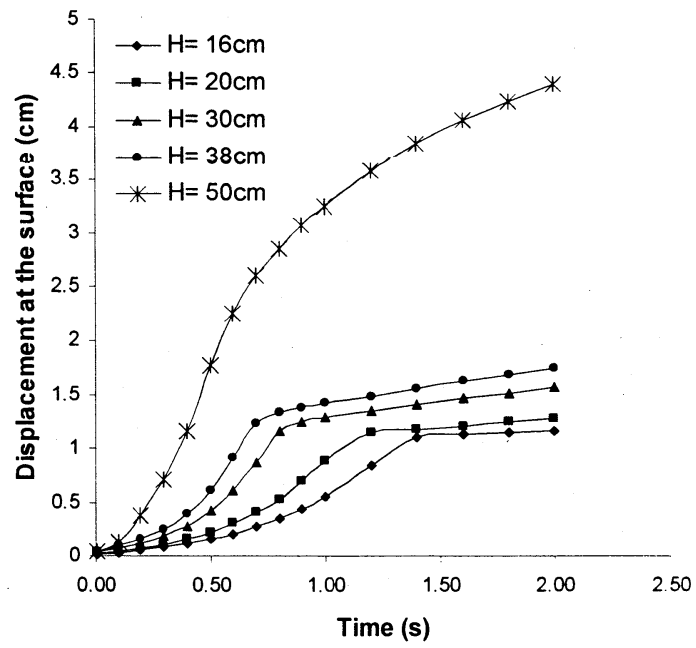

Fig. 14 Time history of the induced displacement for a slope gradient $\theta=3 \%$ 


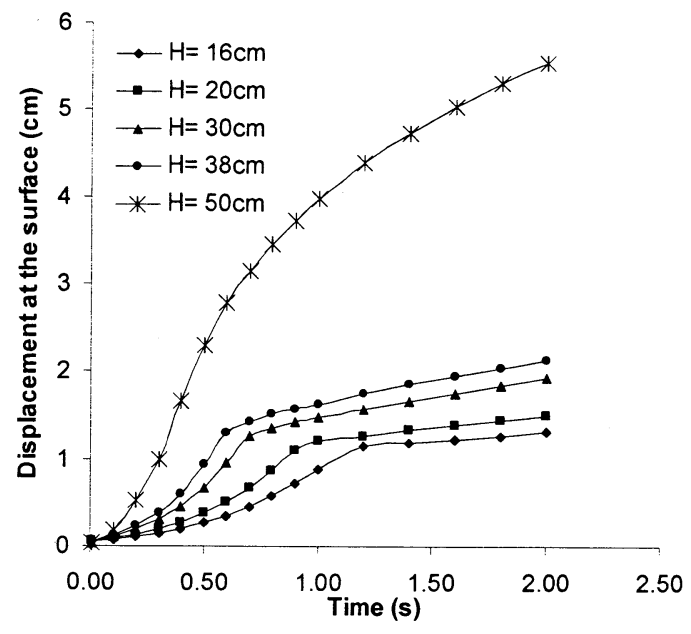

Fig. 15 Time history of the induced displacement for a slope gradient $\theta=5 \%$

\section{Conclusions}

This paper investigates a Lagrangian mesh free particle method using smoothed particle hydrodynamics for liquefaction induced lateral spreading analysis.

Based on post earthquake observations and experimental results, the liquefied soil is considered to behave as a viscous fluid during earthquake shaking. A Bingham type constitutive model was used throughout this study to simulate the behavior of the liquefied soil. The proposed method was first checked to verify results obtained from a shaking table experiment of a lateral spread.

The simulated free surface shape fit with a certain degree of confidence the observed one. Moreover, the general trend of the free surface was clearly observed at the middle where the surface kept almost the same inclination as in the initial configuration. The soil subsided in the upstream and heaved in the downstream due to volume transfer. The time history of the flow velocity was also simulated at the middle cross section at three different locations along the height. The amplitude of the flow velocities was smaller than the observed one, and the flow velocity decreases slowly than the experiment one. This is mainly due to the nature of the constitutive law that does not take into account pore water pressure dissipation and strength recovery.

The distribution of the displacement along the height at the central cross section was also simulated and it was found that the induced displacements are insensitive to the yield viscosity within the simulated range, but sensitive to the minimum undrained shear strength.

In the last part, we carried out numerical simulation to investigate the relationship between the maximum flow velocity, the liquefied soil thickness and the ground gradient slope. Based on numerical results, we observed that the maximum flow velocities increase with increasing layer thickness and slope gradient. We also noticed that the shape of the time history velocity curve at its base narrows with increasing layer thickness. Moreover, the maximum peak velocities flows lay on a curve representing the required time to reach the maximum velocity with respect to the layer thickness.

The relationship between the required time for the flow velocity to reach its maximum was expressed by a power type function in term of liquefied layer thickness. We also expressed the relationship between the flow velocity and the square root of the liquefied layer thickness.

It is worth to mention that this study represents a contribution to analyze liquefaction induced lateral spreading with Lagrangian meshfree particle method using smoothed particle hydrodynamics. It is a promising tool able to handle large displacements. However, it needs improvements to take into account pore water pressure dissipation and strength recovery.

\section{References}

1) Doi, M.and Hamada, M., A summary of Case Studies on Liquefaction-induced Ground displacements, Proc. 4th US-Japan workshop on Earthquake Resistant Design of Lifeline Facilities and Countermeasures against Soil liquefaction, Technical report NCEER 92-0019, 115-129, 1994.

2) Kawakami T., Suemasa N., Hamada H., Sato H.,Katada T., Experimental Study on Mechanical Properties of Liquefied Sand. Proc. 5th US-Japan workshop on Earthquake Resistant Design of Lifeline Facilities and Countermeasures Against Soil liquefaction, Technical report NCEER 94-0026, 285-299, 1994.

3) Towhata I, Material Properties of Liquefied Sand Undergoing Shaking and Large Deformation, US-Japan Workshop on Seismic Disaster Mitigation In Urban Area By Geotechnical Engineering 2002.

4) Yasuda S., Yoshida N., Masuda T., Nagase H., Mine K., Kiku H.,.Stress-strain relationship of liquefied sand, Proc. 1st Int Conf on Earth-Geo Eng, Tokyo 811-816, 1995

5) Aydan O. , Mechanical and numerical modeling of lateral spreading of liquefied soil, Proc.1st Int Conf on Earth-Geo Eng, Tokyo 881-886, 1995

6) Yasuda S., Nagase H., Kiku H., Uchida Y., The mechanism and a simplified procedure for the analysis of permanent ground displacement due to liquefaction, Soil and Foundations, 32(1), 149-160, 1992.

7) Uzuoka R., Yashima A., Kawakami T..,Konrad J.M., Fluid dynamic based prediction of liquefaction induced lateral spreading. Computers and Geotechnics, 22(3/4), 243-282,1998

8) Towhata I., Sasaki Y.,Tokida K., Matsumoto H., Tamari Y., Yamada K. , Prediction of permanent displacement of liquefied ground by means of minimum energy principle. Soil and Foundations, 32(3), 97-116, 1992 
9) Hadush S., Yashima A., Uzuoka R. , Importance of viscous fluid characteristics in liquefaction induced lateral spreading analysis, Computers and Geotechnics, 27, 199-224,2000

10)Maeda K., Sakai M., Development os seepage failure analysis procedure of granular ground with smoothed particle hydrodynamics (SPH) method. Journal of Applied Mechanics, JSCE, 8, 775-786, 2004

11)Monaghan J.J., Simulating free surface flows with SPH. Journal of Computational Physics, 110, 399-406, 1994

12)Towhata I., Vargas-Monge., W, Orense., R.P, Yao, M., Shaking table tests on subgrade reaction of pipe embedded in sandy liquefied subsoil, Soil Dynamics and Earthquake Engineering, 18, 347-361, 1999

13)Hamada, M. and Wakamatsu, K., A study on ground displacement caused by soil liquefaction, Journal of Geotechnical Engineering, JSCE, III-43(596),189-208, 1998
14)Miyajima, M., Kitaura, M., Koike, T., Hasegawa, M., Experimental study on characteristics of liquefied ground flow, Proc.1st Int Conf on Earth-Geo Eng, Tokyo 969- 974, 1995

15)Hamada, H., Sato, H., Kawakami T., A Consideration of the mechanism for liquefaction-related large ground displacement., Proc. 5th US-Japan workshop on Earthquake Resistant Design of Lifeline Facilities and Countermeasures against Soil liquefaction, Technical report NCEER 94-0026, 217-232, 1994.

16)Naili, M., Matsushima, T., Yamada, Y., Numerical simulation of liquefaction induced lateral ground displacements using smoothed particles hydrodynamics, Proc of 2005 convention of the Japan Association for Earthquake Engineering, 400-401, 2005

17)Monaghan J.J., On the problem of penetration in particle methods, Journal of Computational Physics, 82, 1-15, 1989

(Accepted April 15, 2005) 